OPEN ACCESS

Edited by:

Paul G. Higgins,

University of Cologne, Germany

Reviewed by:

Nai-Kei Wong,

Shantou University, China Andrei Nicoli Gebieluca Dabul, University of São Paulo, Brazil

*Correspondence: Hong Zhang schjyk2015@126.com

Specialty section:

This article was submitted to Infectious Diseases - Surveillance,

Prevention and Treatment, a section of the journal

Frontiers in Public Health

Received: 26 October 2020 Accepted: 03 February 2021

Published: 25 February 2021

Citation:

Pan F, Xu Q and Zhang H (2021) Emergence of NDM-5 Producing Carbapenem-Resistant Klebsiella aerogenes in a Pediatric Hospital in Shanghai, China.

Front. Public Health 9:621527. doi: 10.3389/fpubh.2021.621527

\section{Emergence of NDM-5 Producing Carbapenem-Resistant Klebsiella aerogenes in a Pediatric Hospital in Shanghai, China}

\author{
Fen Pan, Qi Xu and Hong Zhang* \\ Department of Clinical Laboratory, Shanghai Children's Hospital, Shanghai Jiaotong University, Shanghai, China
}

Background: Carbapenem-resistant Klebsiella aerogenes (CRKA) has posed a serious threat for clinical anti-infective therapy. However, the molecular characteristics of CRKA in Shanghai are rarely reported.

Objective: This study aimed to investigate the resistance profiles, dissemination mechanism, and molecular characteristics of CRKA strains isolated from children in a pediatric hospital, Shanghai.

Method: Fifty CRKA isolates were collected in 2019. Antimicrobial susceptibility of the strains was determined by broth microdilution method. The $\beta$-lactamases and outer membrane porin genes were characterized by polymerase chain reaction (PCR). Conjugation experiments were performed to determine the transferability of the plasmids. The plasmids were typed based on their incompatibility group using the PCR-based replicon typing method. Multilocus sequence typing (MLST) and enterobacterial repetitive intergenic consensus-PCR (ERIC-PCR) were performed for the genetic relationship.

Results: All CRKA strains showed high level of resistance to cephalosporins and carbapenems, but still susceptible to aminoglycosides, colistin, and tigecycline. Forty five of fifty isolates carried bla genes including bla $a_{\mathrm{CTX}-\mathrm{M}-1}$, bla $\mathrm{T}_{\mathrm{TEM}-1}$, and bla $\mathrm{S}_{\mathrm{SHV}-11}$ being detected. Loss of ompK35 and ompK36 genes were observed in 14\% (7/50) and 28\% (14/50), respectively, with 5 isolates lacking both ompK35 and ompK36. MLST analysis demonstrated that the majority of isolates belonged to ST4 (47/50, 94\%) and ERIC-PCR fingerprinting was performed to identify NDM-5-producing isolates with approximately or more than $80 \%$ similarity levels. Plasmids carrying bla $a_{\mathrm{NDM}-5}$ were successfully transferred to the $E$. coli recipient and plasmid typing showed that IncX3 were the prevalent among CRKA isolates.

Conclusions: Our finding revealed the emergence of NDM-5 producing CRKA belonging to ST4 among children in Shanghai. Further attention should be paid to control the horizontal spread of the Class B carbapenemases like NDM in children.

Keywords: carbapenem-resistant Klebsiella aerogenes, NDM-5, ST4, plasmid, IncX3 


\section{INTRODUCTION}

Klebsiella aerogenes, formerly described as Enterobacter aerogenes, is an opportunistic pathogen associated with a variety of nosocomial infections including pneumonia, sepsis, and urinary tract infection $(1,2)$. Emergence of carbapenem-resistant Enterobacterales (CRE) has posed a serious threat for clinical anti-infective therapy and the main resistant mechanism of CRE is production of carbapenemases, although other mechanisms have been proposed, including overproduction of $\beta$-lactamases, efflux pumps, porin deficiency, and changes in penicillin-binding proteins. Carbapenemases including Klebsiella pneumoniae carbapenemase (KPC), New Delhi metallo- $\beta$-lactamase (NDM), and Imipenemase (IMP) have been identified in $K$. aerogenes (3-5).

NDM carbapenemase, as a newly emerging $\beta$-lactamase, can efficiently hydrolyse a broad range of $\beta$-lactams except aztreonam and has become the most clinically significant due to its increased resistant phenotype and rapid global dissemination. NDM carbapenemases are divided into NDM-1 to NDM-25 variants. To the best of our knowledge, NDM carbapenemases can easily spread among different species of Enterobacterales, with NDM-1 and NDM-5 type being commonly detected. NDM5 carbapenemase was mainly detected in E. coli and rarely in Enterobacterales. However, the prevalence, dissemination mechanism, and clinical characteristics of carbapenem-resistant $K$. aerogenes (CRKA) in Shanghai are still unavailable. In this study, we will focus on CRKA isolated from children in Shanghai, to investigate the resistance profiles and molecular epidemiological characteristics of these strains.

\section{MATERIALS AND METHODS}

\section{Study Design and Bacterial Strains}

This study was retrospectively performed in Shanghai Children's Hospital, which is one of largest pediatric hospitals in China. A total of 50 non-duplicative nosocomial CRKA strains were collected in 2019. All the isolates were identified by matrixassisted laser desorption ionization time of flight (MALDI-TOF) mass spectrometry using MALDI Biotyper (Bruker Daltonik GmbHm, Bremen, Germany). CRKA were defined as strains that were resistant to at least one of the carbapenem antibiotics (ertapenem, meropenem, or imipenem) by Centers for Disease Control and Prevention of USA (https://www.cdc.gov/hai/ organisms/cre/technical-info.html\#Definition). Furthermore, clinical information was obtained from the medical records of each patient. This information included patient demographics, neonatal birth information, brief hospital course, any antibiotic use history of infants, and treatment outcomes, etc.

\section{Antimicrobial Susceptibility Testing}

Antimicrobial susceptibility of all CRKA strains against amikacin, cefuroxime, ceftriaxone, ceftazidime, cefepime, piperacillin-tazobactam, ertapenem, imipenem, meropenem, levofloxacin, trimethoprim-sulfamethoxazole, aztreonam, colistin, and tigecycline were investigated by broth microdilution method. The breakpoints used for interpretation were recommended by the Clinical and Laboratory Standards Institute (CLSI) 2020. The interpretive criterion for tigecycline was based on the breakpoints of the Food and Drug Administration (FDA). Quality control was managed by using E. coli ATCC 25922 and E. coli ATCC 35218.

\section{Detection of Resistance Genes}

DNA of all strains was extracted by boiling centrifugation method. Polymerase chain reaction (PCR) was used to detected carbapenemase genes (bla $a_{\mathrm{NDM}}, b l a_{\mathrm{KPC}}, b l a_{\mathrm{IMP}}, b l a_{\mathrm{VIM}}$, and bla $\left.a_{\mathrm{OXA}-48}\right)$, ESBL genes (bla $\left.a_{\mathrm{CTX}-\mathrm{M}}, b l a_{\mathrm{TEM}}, b l a_{\mathrm{SHV}}\right), \mathrm{AmpC}$ genes (bla $a_{\mathrm{DHA}}, b l a_{\mathrm{CMY}}, b l a_{\mathrm{MOX}}, b l a_{\mathrm{EBC}}$, and $\left.b l a_{\mathrm{AAC}}\right)$ and porin genes (ompK35 and ompK36) as previously described $(6,7)$. Amplicons were sequenced and nucleotide sequences were further analyzed and compared to sequences available at the National Center for Biotechnology Information (NCBI) website (https://blast.ncbi.nlm.nih.gov/Blast.cgi).

\section{Multilocus Sequence Typing}

MLST analysis was performed for the genetic relationship as described previously (8). Seven housekeeping genes including dnaA, fus $A, \operatorname{gyr} B$, leuS, pyrG, $r p l B$, and $r p o B$ were amplified and sequenced for $K$. aerogenes. Alleles and sequence types (STs) were assigned at the PubMLST database (https://pubmlst. org/kaerogenes/). Clonal complexes (CC) of these strains were also analyzed.

\section{Enterobacterial Repetitive Intergenic Consensus-PCR}

Furthermore, the clonal relatedness between NDM-5 producing CRKA isolates were investigated by ERIC-PCR using the primers ERIC-Forward (5'ATGTAAGCTCCTGGGGATTAAC$3^{\prime}$ ) and ERIC-Reverse (5'AAGTAAGGACTGGGG-TGAGCG$\left.3^{\prime}\right)$ (9). Bio-Red Gel Doc system was used to scan gel image and the fingerprinting profiles analysis of these strains was conducted in the BioNumerics software for the similarity dendrogram.

\section{Conjugation and Plasmid Replicon Typing}

To confirm whether the $b l a_{\mathrm{NDM}-5}$ gene was located on plasmids, conjugation transfer experiments were repeatedly carried out by using mixed broth culture method with sodium azide resistant $E$. coli $\mathrm{J} 53$ as the recipient. Potential transconjugants that possessed the $b l a_{\mathrm{NDM}-5}$-bearing plasmid were selected on Mueller-Hinton agar plates containing sodium azide $(180 \mu \mathrm{g} / \mathrm{ml})$ and ertapenem $(1 \mu \mathrm{g} / \mathrm{ml})$. The transconjugants were tested for antimicrobial susceptibilities by broth microdilution for testing the above antibiotics. The colonies grown on the selective medium were identified by detecting bla $a_{\mathrm{NDM}-5}$ gene using PCR. Furthermore, PBRT 2.0 kit-PCR-based replicon typing was used for molecular typing of plasmids of transconjugants (10).

\section{Statistical Analysis}

Antimicrobial resistance data was analyzed by using WHONET 5.6. Other statistical data including quantitative data categorical variables was performed by using SPSS 19.0 software (SPSS Inc., Chicago, IL, USA). A value of $P \leq 0.05$ was considered statistically significant. 


\section{RESULTS}

\section{Clinical Presentation of All Patients}

Among 50 patients, most were located at the neonatal department $(48 / 50,96 \%)$ with only two strains from pediatric intensive care unit (PICU). Most of them are male (78\%, 39/50) with $22 \%$ being female. Most children were $<3$-months-old, with only two children being 5-months-old and 7-months-old, respectively. The median length of hospital stay was 50 days (6-121 days) and the patients developed CRKP colonization or infection an average of 14 days (2-58 days) after admission. Among 48 patients in neonatal unit, the median birth weight was $1300 \mathrm{~g}(770-4100 \mathrm{~g})$ and 33 patients were diagnosed as premature infants.

The most common underlying condition among all patients was pneumonia $(68 \%, 34 / 50)$. Ninety-two percent of patients had a history of invasive procedures, including central venous catheter, intubation/mechanical ventilation, lumbar puncture, and surgery. Most patients have received more than two antibiotics and $\beta$-lactam/ $\beta$-lactamase inhibitor combinations $(74 \% ; 37 / 50)$ and carbapenems $(64 \% ; 32 / 50)$ were the most frequently used antibiotics. Furthermore, a majority of children were improved after treatment during hospitalization and two children were died.

\section{Isolates Information and Antimicrobial Susceptibility of CRKA Isolates}

Among 50 non-duplicated CRKA isolates, 33 (66\%) strains were isolated from sputum, followed by tracheal $(10 / 50,20 \%)$, blood $(3 / 50,6 \%)$, swab $(3 / 50,6 \%)$, and urine $(1 / 50,2 \%)$. As shown in Table 1, 100, 98, and $98 \%$ of isolates were resistant to ertapenem, imipenem, and meropenem, respectively. Furthermore, all strains were resistant to cefuroxime, cefotaxime, ceftriaxone, cefepime, and piperacillin-tazobactam, while all strains were susceptible to amikacin, gentamicin, trimethoprimsulfamethoxazole, and tigecycline. In addition, resistance to levofloxacin was seen in $30 \%$ of isolates in this study.

\section{Distribution of Resistant Genes}

Forty five of fifty isolates carried bla $a_{\mathrm{NDM}-5}$ genes $(45 / 50,90 \%)$, which showed that production of NDM-5 carbapenemase was the main mechanism of $K$. aerogenes resistance to carbapenems. Furthermore, loss of ompK35 and ompK36 genes were also observed in $14 \%(7 / 50)$ and $28 \%(14 / 50)$, respectively, with 5 isolates lacking both $o m p K 35$ and $o m p K 36$. Twelve of 45 NDM5 producing strains examined were deficient in ompK35 or/and ompK36. Among 5 non-NDM-5-producing isolates, ompK35 or/and $o m p K 36$ were detected in four of them. Moreover, all isolates were found to carry at least one ESBL and/or AmpC gene. Specially, bla $a_{\mathrm{CTX}-\mathrm{M}-1}$ group, bla $a_{\mathrm{TEM}-1}, b l a_{\mathrm{SHV}-11}$, and $b l a_{\mathrm{EBC}}$ were identified in $96,96,56$, and $86 \%$, respectively (Table 2 ).

\section{Genetic Relationships}

MLST analysis demonstrated that these 50 isolates were designed into four different STs and most of isolates (47/50, 94\%) belonged to ST4 with allelic profile 3-3-1-4-3-1-2. ST199 and other two new STs were also found in this study. ST4 were identified as
TABLE 1 | Antimicrobial susceptibility profiles of carbapenem-resistant Klebsiella aerogenes isolates.

\begin{tabular}{|c|c|c|c|c|}
\hline \multirow[t]{2}{*}{ Antibiotics } & \multicolumn{2}{|c|}{$\begin{array}{c}\text { Antimicrobial } \\
\text { susceptibility, \% (n) }\end{array}$} & \multicolumn{2}{|c|}{$\operatorname{MIC}(\mu \mathrm{g} / \mathrm{mL})$} \\
\hline & $\mathbf{R}$ & $\mathbf{S}$ & MIC50 & MIC90 \\
\hline Cefuroxime & $100(50)$ & $0(0)$ & $\geq 32$ & $\geq 32$ \\
\hline Cefotaxime & $100(50)$ & $0(0)$ & $\geq 64$ & $\geq 64$ \\
\hline Ceftriaxone & $100(50)$ & $0(0)$ & $\geq 64$ & $\geq 64$ \\
\hline Cefepime & $100(50)$ & $0(0)$ & $\geq 32$ & $\geq 32$ \\
\hline Piperacillin-tazobactam & $100(50)$ & $0(0)$ & $\geq 128 / 2$ & $\geq 128 / 2$ \\
\hline Ertapenem & $100(50)$ & $0(0)$ & $\geq 128$ & $\geq 128$ \\
\hline Imipenem & $98(49)$ & $2(1)$ & $\geq 128$ & $\geq 128$ \\
\hline Meropenem & $98(49)$ & $2(1)$ & $\geq 128$ & $\geq 128$ \\
\hline Amikacin & $0(0)$ & $100(50)$ & $\leq 2$ & $\leq 2$ \\
\hline Levofloxacin & $30.0(15)$ & $2.0(1)$ & 1 & 1 \\
\hline Trimethoprim-sulfamethoxazole & $0(0)$ & $100(50)$ & $\leq 1 / 19$ & $\leq 1 / 19$ \\
\hline Colistin & - & $0(0)$ & $\leq 0.5$ & $\leq 0.5$ \\
\hline Tigecycline & $0(0)$ & $100(50)$ & $\leq 0.5$ & 1 \\
\hline
\end{tabular}

TABLE 2 | Distribution of resistant genes and molecular characteristics of CRKA isolates (No.).

\begin{tabular}{|c|c|c|}
\hline Characteristics & $\begin{array}{l}\text { NDM-5 producing } \\
\text { CRKA }\end{array}$ & $\begin{array}{l}\text { Non-NDM-5 producing } \\
\text { CRKA }\end{array}$ \\
\hline $\begin{array}{l}\text { Other } \\
\beta \text {-lactamase } \\
\text { genes }\end{array}$ & $\begin{array}{l}\text { bla } \\
\text { blateM-M-1 }(45) \\
(26), \text { bla } \\
\text { EBC }(40)\end{array}$ & $\begin{array}{l}\text { blactX }-\mathrm{M}-1 \text { (3), blatEM }-1 \text { (3), } \\
\text { blasHV-11 }(2), b_{\mathrm{EBC}} \text { (3) }\end{array}$ \\
\hline Porin genes & $\begin{array}{l}\text { ompK35(+)+ompK36(+) (33) } \\
\text { ompK35(+)+ompK36(-) } \\
(7) \\
\text { ompK35(-)+ompK36(+) } \\
(2) \\
\text { ompK35(-)+ompK36(-) (3) }\end{array}$ & $\begin{array}{l}\text { ompK35(+)+ompK36(+) (1) } \\
\text { ompK35(+)+ompK36(-) (2) } \\
\text { ompK35(-)+ompK36(+) (2) }\end{array}$ \\
\hline MLST & ST 4(45) & $\begin{array}{l}\text { ST4 (2), ST199 (1), New STs } \\
\text { (2) }\end{array}$ \\
\hline Replicon type & $\operatorname{IncX3~(45)~}$ & / \\
\hline
\end{tabular}

CC1. For molecular typing of NDM-5 producing CRKA isolates, a cluster analysis by ERIC-PCR fingerprinting was performed and thirteen distinct ERIC-PCR types labeled as A-M were identified with approximately or more than $80 \%$ similarity level, which represented that there existed clonal dissemination among neonatal unit or PICU (Figure 1).

\section{Conjugation and Plasmid Replicon Typing Analysis}

Conjugation experiments revealed that potential transconjugants with plasmids were selected and further identified. The E. coli transconjugants exhibited significantly reduced carbapenem susceptibility, and acquired antimicrobial susceptibility pattern similar to those of the donors. In addition, PCR assays demonstrated that all transconjugants harbored the $b l a_{\mathrm{NDM}-5}$ gene and it was successfully transferred from the 45 NDM5-producing CRKA isolates to E. coli J53. According to the 


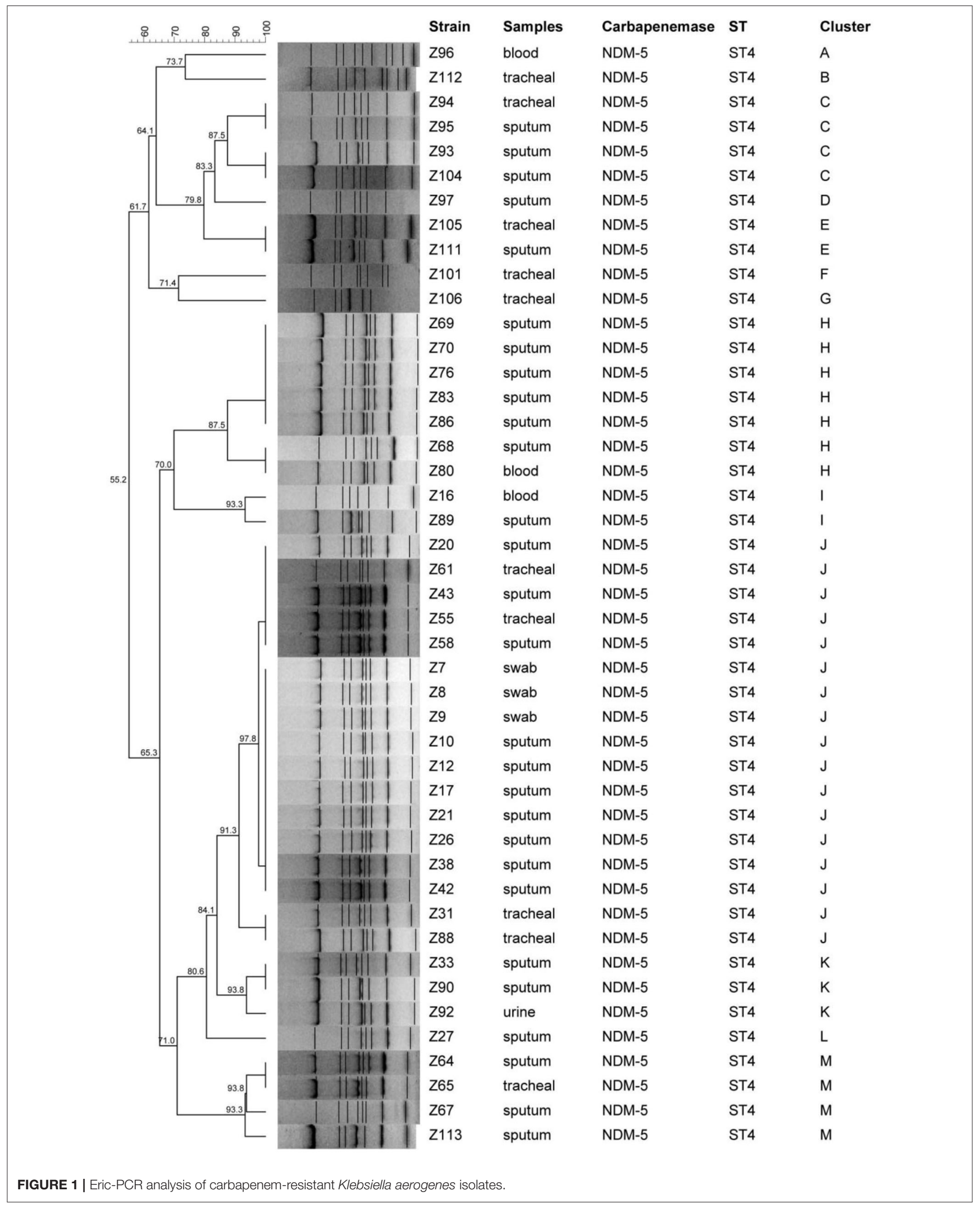


PCR-based plasmid replicon typing results, plasmid types of transconjugants carrying bla $a_{\mathrm{NDM}-5}$ belonged to IncX3 (Table 2).

\section{DISCUSSIONS}

The most common and unequivocal carbapenem resistance mechanism of Enterobacterales involves the production of carbapenemases and this study indicated that NDM-5 was the common carbapenemase in CRKA strains, which was different from other reports conducted in other countries $(3,11)$. Notably, the detection rate for NDM type carrying Enterobacterales has risen in recent years. NDM-5 type carbapenemase, a variant of NDM-1 with two amino acids substitution (Val88Leu and Met154Leu), was firstly identified in E. coli isolate ST648 in United Kingdom from a patient with a previous history of hospitalization in India (12). Then NDM-5 producing strains further spread worldwide, for example, in Europe, United States, Korea, and China, and attracted extensive attention (13-15). Additionally, previous studies showed that NDM-5 carbapenemase has been limited primarily in E. coli and $K$. pneumoniae, but rarely in other species of Enterobacterales, although NDM-5 has been found in Proteus mirabilis (16), Morganella morganii (17), Klebsiella quasipneumoniae (18). Worryingly, NDM-5 producing isolates are also observed in food animals (19) and environment (20) and this phenomenon should be further emphasized.

Despites of the production of carbapenemase, the role of porin deficiency or loss of Omp35 and Omp36 has ever been considered as the main resistant mechanism to carbapenem for Enterobacter including $K$. aerogenes, which was previously assigned as a species of Enterobacter in 1960. In this study, 16 CRKA strains were detected with loss of ompK35 and ompK36 genes and four of 5 non-NDM-5-producing isolates were observed with the loss of ompK35 or/and ompK36 genes. However, recently the viewpoint about the role of porin deficiency in CREA was still controversial (21), with the wide spread of carbapenemase among Enterobacterales. Therefore, it is necessary that we should not neglect the importance of porin deficiency, which maybe the main mechanism of non-carbapenemase-carrying CRE, and extensive studies should be further investigated.

The K. aerogenes MLST scheme was published in December 2017 (22) and only few studies about molecular characteristics of CRKA all over the world were reported. A previous research presented the detailed description of 91 isolates of $K$. aerogenes with whole-genome sequencing reads available on Genbank and the results showed that the prevalent STs of $K$. aerogenes were ST93 and ST4, belonging to CC3 and CC1, respectively (22). Our MLST analysis suggested that ST4 was the predominant ST type in CRKA strains and there was clonal dissemination among neonates, which was further confirmed by Eric-PCR method. In 2017, we have detected four CRKA isolates from children harboring NDM-5 in our hospital but we did not know the ST of theses isolates (23). Whether the previous isolates are correlated to our study is still unknown and further analysis should be conducted. Therefore, this is the first report of ST4 $b l a_{\mathrm{NDM}-5}$-carrying $K$. aerogenes in children in Shanghai.
To the best of our knowledge, mobile genetic elements such as plasmids are independent of the host genome and responsible for horizontal transfer of resistant genes in Enterobacterales. The bla $a_{\mathrm{NDM}-5}$, encoding NDM cabapenemase, was previously identified in IncX 3 and IncFII plasmids $(15,24)$. Here, bla $a_{\mathrm{NDM}-5}$ gene was demonstrated to be located on IncX3 plasmids and the production of bla $a_{\mathrm{NDM}-5}$-harboring CRKA may be the result of the transmission of IncX3 plasmid from other Enterobacterales, since previous studies revealed that the IncX3 plasmids played a significant role in mediating the widespread of the $b a_{\mathrm{NDM}-5}$ gene among Enterobacterales, which have been reported in China (23), India (25), and Nigeria (18). IncX3 belonging to IncXtype plasmids always also bear other $\beta$-lactamase genes (blasHV, $\left.b l a_{\mathrm{TEM}}, b l a_{\mathrm{ampC}}\right)$ and other resistance genes ( $q n r$, sull, and tet) which contribute to resistance to other antibiotics (17).

In conclusion, this study described the emergence of ST4 NDM-5-producing $K$. aerogenes from children in China and the nosocomial infection of these strain among neonates. The present study also provided the evidence about the clonal dissemination of bla $a_{\mathrm{NDM}-5}$ in $K$. aerogenes. Therefore, it is crucial that urgent and effective measures should be taken to monitor and control the further horizontal spread of the Class B carbapenemases like NDM in children.

\section{DATA AVAILABILITY STATEMENT}

The raw data supporting the conclusions of this article will be made available by the authors, without undue reservation.

\section{ETHICS STATEMENT}

The design of the work has been approved by Shanghai Children's Hospital Ethics Committee. This study aimed to obtain the genus and species of bacteria and did not affect the patients. Therefore, our Ethics Committee exempted the patient's informed consent requirements.

\section{AUTHOR CONTRIBUTIONS}

FP and QX conceived and designed the experiments, performed the experiments, and wrote the paper. FP analyzed the data. HZ contributed reagents, materials, and analysis tools. All authors contributed to the article and approved the submitted version.

\section{FUNDING}

This study was supported by the foundation of Shanghai Municipal Key Clinical Specialty (shslczdzk06902), Shanghai Municipal Commission of Health and Family Planning (201940253), and research project of Shang Children's Hospital (2020YGZQ06).

\section{ACKNOWLEDGMENTS}

The authors thank all the patients who contributed their specimens to this study. We also thank the microbiologists and technical staff from Shang Children's hospital. 


\section{REFERENCES}

1. Malek A, McGlynn K, Taffner S, Fine L, Tesini B, Wang J, et al. Next-Generation-Sequencing-Based Hospital Outbreak Investigation Yields Insight into Klebsiella aerogenes Population Structure and Determinants of Carbapenem Resistance and Pathogenicity. Antimicrob Agents Chemother. (2019) 63:e02577-18. doi: 10.1128/AAC.02577-18

2. Davin-Regli A, Pages JM. Enterobacter aerogenes and Enterobacter cloacae; versatile bacterial pathogens confronting antibiotic treatment. Front Microbiol. (2015) 6:392. doi: 10.3389/fmicb.2015.00392

3. Hao M, Shen Z, Ye M, Hu F, Xu X, Yang Y, et al. Outbreak of Klebsiella pneumoniae carbapenemase-producing Klebsiella aerogenes strains in a tertiary hospital in China. Infect Drug Resist. (2019) 12:3283-90. doi: $10.2147 /$ IDR.S221279

4. Shen X, Liu L, Yu J, Cao X, Zhan Q, Guo Y, et al. Coexistence of bla NDM-1 and rmtC on a transferrable plasmid of a Novel ST192 Klebsiella aerogenes clinical isolate. Infect Drug Resist. (2019) 12:3883-91. doi: 10.2147/IDR.S228130

5. Lee JH, Lee $\mathrm{CH}$, Bae IK. Emergence of IMP-4-producing Enterobacter aerogenes clinical isolate. Clin Lab. (2018) 64:1323-6. doi: 10.7754/Clin.Lab.2018.180211

6. Kong Z, Cai R, Cheng C, Zhang C, Kang H, Ma P, et al. First reported nosocomial outbreak of NDM-5-producing Klebsiella pneumoniae in a neonatal unit in China. Infect Drug Resist. (2019) 12:3557-66. doi: 10.2147/IDR.S218945

7. Wassef M, Abdelhaleim M, AbdulRahman E, Ghaith D. The Role of OmpK35, OmpK36 porins, and production of beta-Lactamases on imipenem susceptibility in Klebsiella pneumoniae clinical isolates, Cairo, Egypt. Microb Drug Resist. (2015) 21:577-80. doi: 10.1089/mdr.2014.0226

8. Jolley KA, Bray JE, Maiden MCJ. Open-access bacterial population genomics: BIGSdb software, the PubMLST.org website and their applications. Wellcome Open Res. (2018) 3:124. doi: 10.12688/wellcomeopenres.14826.1

9. Versalovic J, Koeuth T, Lupski JR. Distribution of repetitive DNA sequences in eubacteria and application to fingerprinting of bacterial genomes. Nucleic Acids Res. (1991) 19:6823-31. doi: 10.1093/nar/19.24.6823

10. Carattoli A, Bertini A, Villa L, Falbo V, Hopkins KL, Threlfall EJ. Identification of plasmids by PCR-based replicon typing. J Microbiol Methods. (2005) 63:219-28. doi: 10.1016/j.mimet.2005.03.018

11. Shen Y, Xiao WQ, Gong JM, Pan J, Xu QX. Detection of New Delhi metallobeta-lactamase (encoded by blaNDM-1) in Enterobacter aerogenes in China. J Clin Lab Anal. (2017) 31:e22044. doi: 10.1002/jcla.22044

12. Hornsey M, Phee L, Wareham DW. A novel variant, NDM-5, of the New Delhi metallo-beta-lactamase in a multidrug-resistant Escherichia coli ST648 isolate recovered from a patient in the United Kingdom. Antimicrob Agents Chemother. (2011) 55:5952-4. doi: 10.1128/AAC.05108-11

13. Giufre M, Errico G, Accogli M, Monaco M, Villa L, Distasi MA, et al. Emergence of NDM-5-producing Escherichia coli sequence type 167 clone in Italy. Int J Antimicrob Agents. (2018) 52:76-81. doi: 10.1016/j.ijantimicag.2018.02.020

14. Rojas LJ, Hujer AM, Rudin SD, Wright MS, Domitrovic TN, Marshall SH, et al. NDM-5 and OXA-181 beta-lactamases, a significant threat continues to spread in the Americas. Antimicrob Agents Chemother. (2017) 61:e00454-17. doi: 10.1128/AAC.00454-17

15. Li X, Fu Y, Shen M, Huang D, Du X, Hu Q, et al. Dissemination of blaNDM-5 gene via an IncX3-type plasmid among non-clonal Escherichia coli in China. Antimicrob Resist Infect Control. (2018) 7:59. doi: 10.1186/s13756-018-0349-6

16. Sun L, Xu J, He F. Genomic characterisation of a Proteus mirabilis clinical isolate from China carrying blaNDM-5 on an IncX3 plasmid. $J$ Glob Antimicrob Resist. (2019) 19:317-9. doi: 10.1016/j.jgar.2019.10.025

17. Guo X, Rao Y, Guo L, Xu H, Lv T, Yu X, et al. Detection and genomic characterization of a Morganella morganii isolate from China that produces NDM-5. Front Microbiol. (2019) 10:1156. doi: 10.3389/fmicb.2019.01156

18. Brinkac LM, White R, D'Souza R, Nguyen K, Obaro SK, Fouts DE. Emergence of New Delhi Metallo-beta-Lactamase (NDM-5) in Klebsiella quasipneumoniae from Neonates in a Nigerian Hospital. mSphere. (2019) 4:e00685-18. doi: 10.1128/mSphere.00685-18

19. Hong JS, Song W, Jeong SH. Molecular characteristics of NDM-5-producing Escherichia coli from a cat and a dog in South Korea. Microb Drug Resist. (2020) 26:1005-8. doi: 10.1089/mdr.2019.0382

20. Sekizuka T, Inamine Y, Segawa T, Kuroda M. Characterization of NDM-5and CTX-M-55-coproducing Escherichia coli GSH8M-2 isolated from the effluent of a wastewater treatment plant in Tokyo Bay. Infect Drug Resist. (2019) 12:2243-9. doi: 10.2147/IDR.S215273

21. Hao M, Ye M, Shen Z, Hu F, Yang Y, Wu S, et al. Porin deficiency in carbapenem-resistant Enterobacter aerogenes strains. Microb Drug Resist. (2018) 24:1277-83. doi: 10.1089/mdr.2017.0379

22. Passarelli-Araujo H, Palmeiro JK, Moharana KC, Pedrosa-Silva F, Dalla-Costa LM, Venancio TM. Genomic analysis unveils important aspects of population structure, virulence, and antimicrobial resistance in Klebsiella aerogenes. FEBS J. (2019) 286:3797-810. doi: 10.1111/febs.15005

23. Tian D, Wang B, Zhang H, Pan F, Wang C, Shi Y, et al. Dissemination of the bla NDM-5 Gene via IncX3-Type Plasmid among Enterobacteriaceae in Children. mSphere. (2020) 5:e00699-19. doi: 10.1128/mSphere.00699-19

24. Xu L, Wang P, Cheng J, Qin S, Xie W. Characterization of a novel bla NDM5-harboring IncFII plasmid and an mcr-1-bearing IncI2 plasmid in a single Escherichia coli ST167 clinical isolate. Infect Drug Resist. (2019) 12:511-9. doi: 10.2147/IDR.S192998

25. Krishnaraju M, Kamatchi C, Jha AK, Devasena N, Vennila R, Sumathi G, et al. Complete sequencing of an IncX3 plasmid carrying blaNDM-5 allele reveals an early stage in the dissemination of the blaNDM gene. Indian J Med Microbiol. (2015) 33:30-8. doi: 10.4103/0255-0857.148373

Conflict of Interest: The authors declare that the research was conducted in the absence of any commercial or financial relationships that could be construed as a potential conflict of interest.

Copyright (0) 2021 Pan, Xu and Zhang. This is an open-access article distributed under the terms of the Creative Commons Attribution License (CC BY). The use, distribution or reproduction in other forums is permitted, provided the original author(s) and the copyright owner(s) are credited and that the original publication in this journal is cited, in accordance with accepted academic practice. No use, distribution or reproduction is permitted which does not comply with these terms. 